\title{
Long-Long or Long-Short Range Interactions of Nonlinear Schrödinger Systems in One Space Dimension
}

\author{
Shuang $\operatorname{Lin}^{1}$ and Chunhua $\operatorname{Li}^{1 *}$ \\ ${ }^{1}$ Department of Mathematics, College of Science, Yanbian University, No.977 Gongyuan Road, \\ Yanji City, Jilin Province, 133002, China \\ * Email: sxlch@ybu.edu.cn
}

\begin{abstract}
We consider the initial value problem for systems of one dimensional nonlinear Schrödinger equations with long-long or long-short range interactions. Global existence and time decay of small solutions are presented under mass resonance conditions.
\end{abstract}

Keywords: System of NLS equations; critical, subcritical and supercritical nonlinearities; mass resonance conditions.

2000 Mathematics Subject Classification: 35Q55, 35B40

\section{Introduction and Main Results}

Nonlinear Schrödinger systems are studied extensively from the view points of theory and applications, (see e.g., [1], [5], [20]). In this paper, we consider the Cauchy problem for the following nonlinear Schrödinger system

$$
\left\{\begin{array}{c}
i \partial_{t} v_{1}+\frac{1}{2 m_{1}} \partial_{x}^{2} v_{1}=\lambda_{1}\left|v_{1}\right|^{p_{1}} v_{1}+\mu_{1} \overline{v_{2}} v_{3}^{2} \\
i \partial_{t} v_{2}+\frac{1}{2 m_{2}} \partial_{x}^{2} v_{2}=\lambda_{2}\left|v_{2}\right|^{p_{2}} v_{2}+\mu_{2} \overline{v_{1}} v_{3}^{2} \\
i \partial_{t} v_{3}+\frac{1}{2 m_{3}} \partial_{x}^{2} v_{3}=\lambda_{3}\left|v_{3}\right|^{p_{3}} v_{3}+\mu_{3} v_{1} v_{2} \overline{v_{3}} \\
v_{j}(0, x)=\phi_{j}(x)
\end{array}\right.
$$

in one space dimension, where $x \in \mathbb{R}, t>0, m_{j}$ is a mass of a particle, $v_{j}$ is an unknown function, $p_{j}>0, \lambda_{j}, \mu_{j} \in \mathbb{C} \backslash\{0\}$ for $j=1,2,3$. We assume that the mass resonance condition

$$
m_{1}+m_{2}=2 m_{3}
$$

We define $E=e^{-\frac{i}{2} t|\xi|^{2}}, M=e^{-\frac{i}{2 t}|x|^{2}}, D_{t} \phi=(i t)^{-\frac{1}{2}} \phi\left(\frac{x}{t}\right)$ and $\mathcal{F}$ is the Fourier transform. For $\delta \neq 0$, evolution operator $U_{\delta}(t)$ is written as

$$
U_{\delta}(t)=M^{-\frac{1}{\delta}} D_{\delta t} \mathcal{F} M^{-\frac{1}{\delta}}
$$

We also have

$$
U_{\delta}(-t)=i M^{\frac{1}{\delta}} \mathcal{F}^{-1} E^{\delta} D_{\frac{1}{\delta t}} .
$$

These formulas are used to study asymptotic behavior of solutions to nonlinear Schrödinger equations (see $[10])$.

There is a large amount of studies (see e.g., [2], [22],[25] and [6] ) on the following nonlinear Schrödinger equation

$$
i \partial_{t} u+\frac{1}{2} \partial_{x}^{2} u=|u|^{q} u
$$

in one space dimension, where $x \in \mathbb{R}, q>0$. When $0<q \leq 2$, nonexistence of asymptotic free solutions to (1.3) was studied in [2]. On the other hand, existence of asymptotic free solutions to (1.3) was studied in [25], if $q>2$ holds. Therefore $q=2$ is regarded as the borderline of the short range interactions and long range ones in (1.3). The initial value problem for nonlinear Schrödinger equation

$$
i \partial_{t} u+\frac{1}{2} \partial_{x}^{2} u=\lambda|u|^{q} u
$$


in one space dimension, where $x \in \mathbb{R}, t>0, \lambda \in \mathbb{C} \backslash\{0\}, q=2$, was studied in [24] for small initial data in the case of $\operatorname{Im} \lambda<0$. If $q<2$ and $q$ is close to 2, (1.4) was investigated in [15] under the dissipative condition such that $\operatorname{Im} \lambda<0$ with the smallness conditions on the initial data, and in [16] and [12] under the strong dissipative conditions such that $\operatorname{Im} \lambda<0$ and $|\operatorname{Im} \lambda| \geq \frac{q}{2 \sqrt{q+1}}|\operatorname{Re} \lambda|$ without smallness conditions on the initial data. In these cases, nonlinear effects of the equation (1.4) are considered as dissipative ones. In [22], the final state problem for nonlinear Schrödinger equation with the nonlinear interaction term

$$
i \partial_{t} u+\frac{1}{2} \partial_{x}^{2} u=\lambda|u|^{2} u+\mu|u|^{q} u
$$

in one space dimension, where $x \in \mathbb{R}, t>0, u$ is an unknown function, $q>2, \lambda \in \mathbb{R} \backslash\{0\}, \mu \in \mathbb{R}$, was considered and modified scattering operator was constructed. In this case, the first term of the equation (1.5) acts as the long range effect. On the other hand, the initial value problem for the equation (1.5) was studied in [6] and the existence of modified scattering states was shown. After these works, scattering problem for the similar equations was developed by many authors ( see e.g., [3] and [7]), and asymptotic behavior of the solutions are well known. Some derivative nonlinear Schrödinger equations were also considered (see e.g., [19] and [23]). For the Schrödinger systems with long range interactions, asymptotic behavior of solutions was known on the final state problem (see, e.g., [21], [9]). There are some results on the initial value problem for the Schrödinger systems (see e.g., [8], [13], [14] and [18]) and time decay estimates of the solutions were studied in some critical cases. The existence of ground states for some nonlinear Schrödinger systems was investigated in [11]. To the authors' knowledge, there is no previous research about the system of nonlinear Schrödinger equations with critical and supcritical nonlinearities or critical and subcritical nonlinearities.

From the previous works (see e.g., [8], [13] and [14] ), it is known that the asymptotic behavior or time decay of solutions to (1.1) is determined by solutions to the system of ordinary differential equations

$$
\left\{\begin{array}{c}
i \partial_{t} w_{1}=\lambda_{1} t^{-\frac{p_{1}}{2}}\left|w_{1}\right|^{p_{1}} w_{1}+\mu_{1} t^{-1} \overline{w_{2}} w_{3}^{2} \\
i \partial_{t} w_{2}=\lambda_{2} t^{-\frac{p_{2}}{2}}\left|w_{2}\right|^{p_{2}} w_{2}+\mu_{2} t^{-1} \overline{w_{1}} w_{3}^{2} \\
i \partial_{t} w_{3}=\lambda_{3} t^{-\frac{p_{3}}{2}}\left|w_{3}\right|^{p_{3}} w_{3}+\mu_{3} t^{-1} w_{1} w_{2} \overline{w_{3}}
\end{array}\right.
$$

since we have from $(1.1)$

$$
\left\{\begin{array}{c}
i \partial_{t} u_{1}=\lambda_{1} t^{-\frac{p_{1}}{2}}\left|u_{1}\right|^{p_{1}} u_{1}+\mu_{1} t^{-1} \overline{u_{2}} u_{3}^{2}+R_{1}, \\
i \partial_{t} u_{2}=\lambda_{2} t^{-\frac{p_{2}}{2}}\left|u_{2}\right|^{p_{2}} u_{2}+\mu_{2} t^{-1} \overline{u_{1}} u_{3}^{2}+R_{2}, \\
i \partial_{t} u_{3}=\lambda_{3} t^{-\frac{p_{3}}{2}}\left|u_{3}\right|^{p_{3}} u_{3}+\mu_{3} t^{-1} u_{1} u_{2} \overline{u_{3}}+R_{3}
\end{array}\right.
$$

where

$$
u_{j}=D_{\frac{1}{m_{j}}} \mathcal{F} U_{\frac{1}{m_{j}}}(-t) v_{j}
$$

and $R_{j}$ are the remainder terms under some conditions on $p_{j}, \lambda_{j}, \mu_{j}$ for $j=1,2,3$. We note here that we do not know the asymptotic behavior of solutions of the system (1.6). This is the reason why asymptotic behavior of solutions to our problems is not derived except the time decay. When $p_{j}=2$ for $j=1,2,3$, the system (1.1) becomes

$$
\left\{\begin{array}{c}
i \partial_{t} v_{1}+\frac{1}{2 m_{1}} \partial_{x}^{2} v_{1}=\lambda_{1}\left|v_{1}\right|^{2} v_{1}+\mu_{1} \overline{v_{2}} v_{3}^{2} \\
i \partial_{t} v_{2}+\frac{1}{2 m_{2}} \partial_{x}^{2} v_{2}=\lambda_{2}\left|v_{2}\right|^{2} v_{2}+\mu_{2} \overline{v_{1}} v_{3}^{2} \\
i \partial_{t} v_{3}+\frac{1}{2 m_{3}} \partial_{x}^{2} v_{3}=\lambda_{3}\left|v_{3}\right|^{2} v_{3}+\mu_{3} v_{1} v_{2} \overline{v_{3}} \\
v_{j}(0, x)=\phi_{j}(x) .
\end{array}\right.
$$

Therefore the system (1.7) is a special situation of the system (1.1). Global existence of small solutions to the system (1.7) was studied in [14] on some situations of $\lambda_{j}, \mu_{j}$ for $j=1,2,3$. A similar system in two space dimensions

$$
\left\{\begin{array}{l}
i \partial_{t} v_{1}+\frac{1}{2 m_{1}} \Delta v_{1}=\lambda_{1}\left|v_{1}\right| v_{1}+\mu_{1} \overline{v_{2}} v_{3} \\
i \partial_{t} v_{2}+\frac{1}{2 m_{2}} \Delta v_{2}=\lambda_{2}\left|v_{2}\right| v_{2}+\mu_{2} \overline{v_{1}} v_{3} \\
i \partial_{t} v_{3}+\frac{1}{2 m_{3}} \Delta v_{3}=\lambda_{3}\left|v_{3}\right| v_{3}+\mu_{3} v_{1} v_{2} \\
v_{j}(0, x)=\phi_{j}(x)
\end{array}\right.
$$


was first studied in [13]. The author of [14] treats (1.7) by using the similar techniques as [13]. In [14], two theorems are presented. One of them says that if the estimate

$$
\frac{d}{d t} \sum_{j=1}^{3}\left|u_{j}\right| \leq C \sum_{j=1}^{3}\left|R_{j}\right|
$$

holds, where $u_{j}=D_{\frac{1}{m_{j}}} \mathcal{F} U_{\frac{1}{m_{j}}}(-t) v_{j}$, then (1.7) has global small solutions with time decay such that $\|v(t)\|_{\mathbf{L}^{\infty}} \leq C t^{-\frac{1}{2}}$ for $t \geq 1$. Another one of them says that if there exists a positive constant $k$ such that

$$
\frac{d}{d t} \sum_{j=1}^{3}\left|u_{j}\right|+k t^{-1} \sum_{j=1}^{3}\left|u_{j}\right|^{3} \leq C \sum_{j=1}^{3}\left|R_{j}\right|
$$

holds, where $u_{j}=D_{\frac{1}{m_{j}}} \mathcal{F} U_{\frac{1}{m_{j}}}(-t) v_{j}$, then global small solution $v(t)$ to $(1.7)$ exists and we have the time decay such that $\|v(t)\|_{\mathbf{L}^{\infty}} \leq C t^{-\frac{1}{2}}(\log (1+t))^{-\frac{1}{2}}$ for $t \geq 0$. We assume that there exist some positive constants $k_{1}, k_{2}, k_{3}>0$ such that

$$
k_{1} \mu_{1}+k_{2} \mu_{2}=k_{3} \overline{\mu_{3}}
$$

and

$$
\operatorname{Im} \lambda_{j} \leq 0
$$

for $j=1,2,3$. Then we have (1.9). In addition to (1.11), we assume that

$$
\operatorname{Im} \lambda_{j}<0
$$

for $j=1,2,3$. Then we have (1.10). The main idea to get time decay of solutions as $\|v(t)\|_{\mathbf{L}^{\infty} \leq} \leq$ $C t^{-\frac{1}{2}}(\log (1+t))^{-\frac{1}{2}}$ for $t \geq 0$ in [14] can be seen in [13]. We consider the following generalized nonlinear Schrödinger system

$$
\left\{\begin{array}{c}
i \partial_{t} v_{1}+\frac{1}{2 m_{1}} \partial_{x}^{2} v_{1}=\lambda_{1}\left|v_{1}\right|^{p_{1}} v_{1}+G_{1}\left(v_{1}, v_{2}, v_{3}\right), \\
i \partial_{t} v_{2}+\frac{1}{2 m_{2}} \partial_{x}^{2} v_{2}=\lambda_{2}\left|v_{2}\right|^{p_{2}} v_{2}+G_{2}\left(v_{1}, v_{2}, v_{3}\right), \\
i \partial_{t} v_{3}+\frac{1}{2 m_{3}} \partial_{x}^{2} v_{3}=\lambda_{3}\left|v_{3}\right|^{p_{3}} v_{3}+G_{3}\left(v_{1}, v_{2}, v_{3}\right), \\
v_{j}(0, x)=\phi_{j}(x),
\end{array}\right.
$$

in one space dimension, where $x \in \mathbb{R}, t>0, m_{j}$ is a mass of a particle, $v_{j}$ is an unknown function, $p_{j}>0, \lambda_{j} \in \mathbb{C} \backslash\{0\}$, and $G_{j}\left(v_{1}, v_{2}, v_{3}\right)$ is a cubic nonlinear term with the following form

$$
G_{j}\left(v_{1}, v_{2}, v_{3}\right)=\sum_{1 \leq m \leq n \leq l \leq 6} \mu_{m, n, l}^{j} v_{m} v_{n} v_{l}
$$

for $j=1,2,3, v_{m}, v_{n}, v_{l}$ are elements of the set

$$
A=\left\{v_{1}, v_{2}, v_{3}, \overline{v_{1}}, \overline{v_{2}}, \overline{v_{3}}\right\}=\left\{v_{1}, v_{2}, v_{3}, v_{4}, v_{5}, v_{6}\right\}
$$

and $\mu_{m, n, l}^{j} \in \mathbb{C} \backslash\{0\}$. We assume the gauge condition

$$
G_{j}\left(v_{1}, v_{2}, v_{3}\right)=e^{i m_{j} \theta} G_{j}\left(e^{-i m_{1} \theta} v_{1}, e^{-i m_{2} \theta} v_{2}, e^{-i m_{3} \theta} v_{3}\right)
$$

for any $\theta \in \mathbb{R}$. Then we obtain that the system (1.1) with the condition (1.2) is a special case of the generalized system satisfying the gauge condition above. We can solve the initial value problem of the generalized Schrödinger system with the gauge condition by using the similar method as this paper.

In this paper, we consider the problem (1.1) under different situations on $\lambda_{j}, \mu_{j}$ for small initial data in the function space $\mathbf{H}^{0, \frac{1}{2}+s}(\mathbb{R}) \cap \mathbf{H}^{\frac{1}{2}+s, 0}(\mathbb{R})$, where $\frac{1}{2}<s<1$. In particular, $\mathbf{L}^{\infty}(\mathbb{R})-$ time decay of solutions to (1.1) is obtained. We consider (1.1) under the long-long and long-short range interactions, respectively. We treat the case of long-short range interactions (Theorem 1.1) by using the similar method as [8]. The results on long-long range interactions are new. We consider two situations of long-long range interactions. One of our results below (Theorem 1.2) says that if $\operatorname{Im} \lambda_{j}<0$ and $\left|\operatorname{Im} \lambda_{j}\right|$ is large enough, 
then the first terms of the right hand sides of (1.1) are considered as strong dissipation terms when $p_{j}<2$ for $j=1,2,3$ and the second terms of the right hand sides of (1.1) will be remainder ones. However if $\left|\operatorname{Im} \lambda_{j}\right|$ is not large enough, then our result (Theorem 1.3) says that we can not find which ones will be the main terms. Therefore we assume that (1.11) holds in Theorem 1.3 to make the second terms of the right hand sides of (1.1) be negligeable.

To state our results, we introduce the function spaces. Let $\mathbf{L}^{p}(\mathbb{R})$ denote the usual Lebesgue space with the norm

if $1 \leq p<\infty$ and

$$
\|\phi\|_{\mathbf{L}^{p}(\mathbb{R})}=\left(\int_{\mathbb{R}}|\phi(x)|^{p} d x\right)^{\frac{1}{p}}
$$

$$
\|\phi\|_{\mathbf{L}^{\infty}(\mathbb{R})}=\underset{x \in \mathbb{R}}{\operatorname{ess.sup}}|\phi(x)| .
$$

For $m, r \in \mathbb{R}$ and $1 \leq p \leq \infty$, weighted Sobolev space $\mathbf{H}_{p}^{m, r}(\mathbb{R})$ is defined by

$$
\mathbf{H}_{p}^{m, r}(\mathbb{R})=\left\{f \in \mathbf{L}^{p}(\mathbb{R}) ;\|f\|_{\mathbf{H}_{p}^{m, r}(\mathbb{R})}=\left\|\left(1-\partial_{x}^{2}\right)^{\frac{m}{2}}\left(1+|x|^{2}\right)^{\frac{r}{2}} f\right\|_{\mathbf{L}^{p}(\mathbb{R})}<\infty\right\} .
$$

We write $\|f\|_{\mathbf{L}^{2}(\mathbb{R})}=\|f\|,\|f\|_{\mathbf{L}^{\infty}(\mathbb{R})}=\|f\|_{\mathbf{L}^{\infty}}, \mathbf{H}_{2}^{m, r}(\mathbb{R})=\mathbf{H}^{m, r}$ and $\mathbf{H}^{m, 0}(\mathbb{R})=\mathbf{H}^{m}$ for simplicity. We denote by the same letter $C$ various positive constants.

We define the fractional derivatives of $J_{\frac{1}{m}}=U_{\frac{1}{m}}(t) x U_{\frac{1}{m}}(-t)$ as

$$
\left|J_{\frac{1}{m}}\right|^{\gamma}(t)=U_{\frac{1}{m}}(t)|x|^{\gamma} U_{\frac{1}{m}}(-t) \text {, }
$$

where $\gamma \geq 0$. From [10], We have $\left|J_{\frac{1}{m}}\right|^{\gamma}(t)=M^{-m}\left(-\frac{t^{2}}{m^{2}} \Delta\right)^{\frac{\gamma}{2}} M^{m}$. We also have commutation relations with $\left|J_{\frac{1}{m}}\right|^{\gamma}$ and $L_{\frac{1}{m}}=i \partial_{t}+\frac{1}{2 m} \partial_{x}^{2}$ such that

$$
\left[L_{\frac{1}{m}},\left|J_{\frac{1}{m}}\right|^{\gamma}\right]=0
$$

First we state our result for $p_{j}>2$ for $j=1,2,3$. In this case, the first terms and the second terms on the right hand sides of (1.1) are considered as short and long range interactions, respectively. Therefore the first terms are regarded as remainder ones of the system (1.1).

Theorem 1.1 We assume that (1.2) holds, $p_{j}>2$ for $j=1,2,3$ and there exist some positive constants $k_{1}, k_{2}, k_{3}>0$ such that (1.11) holds. Let $\phi_{j} \in \mathbf{H}^{0, \frac{1}{2}+s} \cap \mathbf{H}^{\frac{1}{2}+s}, \sum_{j=1}^{3}\left\|\phi_{j}\right\|_{\mathbf{H}^{0, \frac{1}{2}+s} \cap \mathbf{H}^{\frac{1}{2}+s}} \leq \varepsilon$, where $\varepsilon$ is sufficiently small, and $\frac{1}{2}<s<1$. Then there exists a unique global solution $\left(v_{j}(t)\right)_{j=1,2,3} \in$ $\mathbf{C}\left([0, \infty) ; \mathbf{H}^{0, \frac{1}{2}+s} \cap \mathbf{H}^{\frac{1}{2}+s}\right)$ to the system (1.1). Moreover, we have the following time decay estimate

$$
\left\|v_{j}(t)\right\|_{\mathbf{L}^{\infty}} \leq C(1+t)^{-\frac{1}{2}}
$$

for $t \geq 0$ and $j=1,2,3$.

Next result says the case $0<p_{j}<2$ for $j=1,2,3$, namely long-long interactions. In this case, second terms on the right hand sides of (1.1) are considered as remainder terms under the condition (1.13) with a strong dissipative condition such that

$$
\frac{1}{\varepsilon \sqrt{\varepsilon}} \leq\left|\operatorname{Im} \lambda_{j}\right|
$$

Therefore we do not need to assume (1.11). Time decay of solutions of (1.1) is still open problem if we do not assume (1.13) and (1.14).

Theorem 1.2 We assume that (1.2), (1.13) and (1.14) hold, $p_{j}=2-\sqrt{\varepsilon}, \phi_{j} \in \mathbf{H}^{0, \frac{1}{2}+s} \cap \mathbf{H}^{\frac{1}{2}+s}$ for $j=1,2,3, \sum_{j=1}^{3}\left\|\phi_{j}\right\|_{\mathbf{H}^{0, \frac{1}{2}+s} \cap \mathbf{H}^{\frac{1}{2}+s}} \leq \varepsilon$, where $\varepsilon$ is sufficiently small, and $\frac{1}{2}<s<1$. Then there exists a unique global solution

$$
\left(v_{j}(t)\right)_{j=1,2,3} \in \mathbf{C}\left([0, \infty) ; \mathbf{H}^{0, \frac{1}{2}+s} \cap \mathbf{H}^{\frac{1}{2}+s}\right)
$$


to the system (1.1). Moreover, we have the following time decay estimate

$$
\left\|v_{j}(t)\right\|_{\mathbf{L}^{\infty}} \leq C(1+t)^{-\frac{1}{p_{j}}}
$$

for $t \geq 0$ and $j=1,2,3$.

If we do not assume (1.14), namely $\left|\operatorname{Im} \lambda_{j}\right| \leq C$, then it seems that dissipation property of the first terms on the right hand sides of (1.1) do not control the time decay of second terms. In this case we use the dispersive condition (1.11) on the second terms.

Theorem 1.3 We assume that (1.2), (1.13) and there exist some positive constants $k_{1}, k_{2}, k_{3}>0$ such that (1.11) holds. Let $p_{j}=2-\varepsilon^{p_{j}}, \phi_{j} \in \mathbf{H}^{0, \frac{1}{2}+s} \cap \mathbf{H}^{\frac{1}{2}+s}$ for $j=1,2,3, \sum_{j=1}^{3}\left\|\phi_{j}\right\|_{\mathbf{H}^{0, \frac{1}{2}+s} \cap \mathbf{H}^{\frac{1}{2}+s}} \leq \varepsilon$, where $\varepsilon$ is sufficiently small, and $\frac{1}{2}<s<1$. Then there exists a unique global solution

$$
\left(v_{j}(t)\right)_{j=1,2,3} \in \mathbf{C}\left([0, \infty) ; \mathbf{H}^{0, \frac{1}{2}+s} \cap \mathbf{H}^{\frac{1}{2}+s}\right)
$$

to the system (1.1). Moreover, we have the following time decay estimate

$$
\left\|v_{j}(t)\right\|_{\mathbf{L}^{\infty}} \leq C(1+t)^{-\frac{1}{p_{j}}}
$$

for $t \geq 0$ and $j=1,2,3$.

\section{Proof of Theorem 1.1}

We define

$$
\|v\|_{X_{T}}=\sup _{t \in[0, T]} \sum_{j=1}^{3}\left((1+t)^{-\sqrt{\varepsilon}}\left\|U_{\frac{1}{m_{j}}}(-t) v_{j}\right\|_{\mathbf{H}^{0, \frac{1}{2}+s}}+\left\|D_{\frac{1}{m_{j}}} \mathcal{F} U_{\frac{1}{m_{j}}}(-t) v_{j}\right\|_{\mathbf{L}^{\infty}}\right),
$$

where $\frac{1}{2}<s<1, T>0$ and $\varepsilon>0$ is sufficiently small.

Lemma 2.1 We assume that assumptions in Theorem 1.1 hold. Then the system (1.1) has a unique pair of solution $v=\left(v_{j}\right)_{j=1,2,3} \in X_{T}$ such that

$$
\|v\|_{X_{T}} \leq 2 \varepsilon
$$

Local existence of solutions to (1.1) is obtained by a standard method, (see, e.g., [4]). Therefore, we prove global existence and time decay estimates of solutions to (1.1). By the local existence result, we may assume that

$$
\sum_{j=1}^{3}\left(\left\|U_{\frac{1}{m_{j}}}(-1) v_{j}(1)\right\|_{\mathbf{H}^{0, \frac{1}{2}+s}}+\left\|D_{\frac{1}{m_{j}}} \mathcal{F} U_{\frac{1}{m_{j}}}(-1) v_{j}(1)\right\|_{\mathbf{L}^{\infty}}\right) \leq 2 \varepsilon
$$

We prove that for any $T$, the estimate

$$
\sup _{t \in[1, T]} \sum_{j=1}^{3}\left(t^{-\sqrt{\varepsilon}}\left\|U_{\frac{1}{m_{j}}}(-t) v_{j}\right\|_{\mathbf{H}^{0, \frac{1}{2}+s}}+\left\|D_{\frac{1}{m_{j}}} \mathcal{F} U_{\frac{1}{m_{j}}}(-t) v_{j}\right\|_{\mathbf{L}^{\infty}}\right)<\varepsilon^{\frac{2}{3}}
$$

holds. By the contradiction, we assume that there exists a time $T$ such that

$$
\sup _{t \in[1, T]} \sum_{j=1}^{3}\left(t^{-\sqrt{\varepsilon}}\left\|U_{\frac{1}{m_{j}}}(-t) v_{j}\right\|_{\mathbf{H}^{0, \frac{1}{2}+s}}+\left\|D_{\frac{1}{m_{j}}} \mathcal{F} U_{\frac{1}{m_{j}}}(-t) v_{j}\right\|_{\mathbf{L}}\right) \leq \varepsilon^{\frac{2}{3}}
$$


Let

$$
\begin{aligned}
& F_{1}:=F_{1}\left(v_{1}, v_{2}, v_{3}\right)=\mu_{1} \overline{v_{2}} v_{3}^{2}, \\
& F_{2}:=F_{2}\left(v_{1}, v_{2}, v_{3}\right)=\mu_{2} \overline{v_{1}} v_{3}^{2}, \\
& F_{3}:=F_{3}\left(v_{1}, v_{2}, v_{3}\right)=\mu_{3} v_{1} v_{2} \overline{v_{3}} .
\end{aligned}
$$

We multiply both sides of (1.1) by $D_{\frac{1}{m_{j}}} \mathcal{F} U_{\frac{1}{m_{j}}}(-t)$ to get

$$
i \partial_{t} u_{j}=\lambda_{j} D_{\frac{1}{m_{j}}} \mathcal{F} U_{\frac{1}{m_{j}}}(-t)\left|v_{j}\right|^{p_{j}} v_{j}+D_{\frac{1}{m_{j}}} \mathcal{F} U_{\frac{1}{m_{j}}}(-t) F_{j}
$$

where $u_{j}=D_{\frac{1}{m_{j}}} \mathcal{F} U_{\frac{1}{m_{j}}}(-t) v_{j}$. Then we have by using the factorization formula $\mathcal{F} U_{\frac{1}{m_{j}}}(-t)=i \mathcal{M}_{m_{j}} E^{\frac{1}{m_{j}}} D_{\frac{m_{j}}{t}}$ with $\mathcal{M}_{m_{j}}=\mathcal{F} M^{m_{j}} \mathcal{F}^{-1}$ to find that

$$
D_{\frac{1}{m_{j}}} \mathcal{F} U_{\frac{1}{m_{j}}}(-t)\left(\lambda_{j}\left|v_{j}\right|^{p_{j}} v_{j}\right)=\lambda_{j} t^{-\frac{p_{j}}{2}}\left|u_{j}\right|^{p_{j}} u_{j}+D_{\frac{1}{m_{j}}} \sum_{h=1}^{2} R_{h, j},
$$

where

$$
\begin{aligned}
R_{1 j}= & \lambda_{j}\left(\frac{i t}{m_{j}}\right)^{-\frac{p_{j}}{2}}\left(\left|\mathcal{F} M^{-m_{j}} U_{\frac{1}{m_{j}}}(-t) v_{j}\right|^{p_{j}} \mathcal{F} M^{-m_{j}} U_{\frac{1}{m_{j}}}(-t) v_{j}\right. \\
& \left.-\left|\mathcal{F} U_{\frac{1}{m_{j}}}(-t) v_{j}\right|^{p_{j}} \mathcal{F} U_{\frac{1}{m_{j}}}(-t) v_{j}\right)
\end{aligned}
$$

and

$$
R_{2 j}=\lambda_{j}\left(\frac{i t}{m_{j}}\right)^{-\frac{p_{j}}{2}} \mathcal{F}\left(M^{m_{j}}-1\right) \mathcal{F}^{-1}\left|\mathcal{F} M^{-m_{j}} U_{\frac{1}{m_{j}}}(-t) v_{j}\right|^{p_{j}} \mathcal{F} M^{-m_{j}} U_{\frac{1}{m_{j}}}(-t) v_{j} .
$$

In the same way as the proof in [17], we have by the resonance gauge condition (1.2)

$$
\begin{aligned}
& D_{\frac{1}{m_{j}}} \mathcal{F} U_{\frac{1}{m_{j}}}(-t) F_{j}\left(v_{1}, v_{2}, v_{3}\right) \\
= & \frac{1}{t} F_{j}\left(u_{1}, u_{2},, u_{3}\right)+D_{\frac{1}{m_{j}}} \sum_{h=3}^{4} R_{h, j},
\end{aligned}
$$

where

$$
\begin{aligned}
& R_{3, j} \\
= & i^{\frac{1}{2}}\left(\mathcal{M}_{m_{j}}-I\right) \frac{m_{j}}{t} F_{j}\left(D_{\frac{m_{j}}{m_{1}}} \mathcal{M}_{m_{1}}^{-1} D_{\frac{1}{m_{1}}}^{-1} u_{1}, D_{\frac{m_{j}}{m_{2}}} \mathcal{M}_{m_{2}}^{-1} D_{\frac{1}{m_{2}}}^{-1} u_{2}, D_{\frac{m_{j}}{m_{3}}} \mathcal{M}_{m_{3}}^{-1} D_{\frac{1}{m_{3}}}^{-1} u_{3}\right)
\end{aligned}
$$

and

$$
\begin{aligned}
& R_{4, j} \\
= & i^{\frac{1}{2}} \frac{m_{j}}{t} F_{j}\left(D_{\frac{m_{j}}{m_{1}}} \mathcal{M}_{m_{1}}^{-1} D_{\frac{1}{m_{1}}}^{-1} u_{1}, D_{\frac{m_{j}}{m_{2}}} \mathcal{M}_{m_{2}}^{-1} D_{\frac{1}{m_{2}}}^{-1} u_{2}, D_{\frac{m_{j}}{m_{3}}} \mathcal{M}_{m_{3}}^{-1} D_{\frac{1}{m_{3}}}^{-1} u_{3}\right) \\
& -i^{\frac{1}{2}} \frac{m_{j}}{t} F_{j}\left(D_{\frac{m_{j}}{m_{1}}} D_{\frac{1}{m_{1}}}^{-1} u_{1}, D_{\frac{m_{j}}{m_{2}}} D_{\frac{1}{m_{2}}}^{-1} u_{2}, D_{\frac{m_{j}}{m_{3}}} D_{\frac{1}{m_{3}}}^{-1} u_{3}\right) .
\end{aligned}
$$

Therefore we have

$$
i \partial_{t} u_{j}=\lambda_{j} t^{-\frac{p_{j}}{2}}\left|u_{j}\right|^{p_{j}} u_{j}+\frac{1}{t} F_{j}\left(u_{1}, u_{2}, u_{3}\right)+D_{\frac{1}{m_{j}}} \sum_{h=1}^{4} R_{h, j} .
$$

In what follows, we only consider the case $t \geq 1$.

To prove our main results, we first show 
Lemma 2.2 Let $\left(v_{j}\right)_{j=1,2,3}$ be a solution of the system (1.1) satisfying (2.1) and $\frac{1}{2}<s<1$, then we have

$$
\sum_{h=1}^{2}\left\|R_{h, j}\right\|_{\mathbf{L}^{\infty}} \leq C \varepsilon^{\frac{2}{3}\left(p_{j}+1\right)} t^{-\frac{p_{j}}{2}-\frac{s}{2}+\sqrt{\varepsilon}\left(p_{j}+1\right)}
$$

and

$$
\sum_{h=3}^{4}\left\|R_{h, j}\right\|_{\mathbf{L}^{\infty}} \leq C \varepsilon^{2} t^{-1-\frac{s}{2}+3 \sqrt{\varepsilon}}
$$

for $t \in[1, T]$ and $j=1,2,3$.

Proof By using the similar method as Lemma 2.1 in [12], we have

$$
\sum_{h=1}^{2}\left\|R_{h, j}\right\|_{\mathbf{L}^{\infty}} \leq C t^{-\frac{p_{j}}{2}-\frac{s}{2}} \sum_{j=1}^{3}\left\|U_{\frac{1}{m_{j}}}(-t) v_{j}\right\|_{\mathbf{H}^{0, \frac{1}{2}+s}}^{p_{j}+1} .
$$

Now we consider $\sum_{h=3}^{4}\left\|R_{h, j}\right\|_{\mathbf{L}^{\infty}}$. By the Sobolev inequality, we have

$$
\|f\|_{\mathbf{L}^{\infty}} \leq C\|f\|_{\mathbf{H}^{\frac{1}{2}+s}}^{\frac{1}{1+2 s}}\|f\|_{\mathbf{L}^{2}}^{\frac{2 s}{1+2 s}}
$$

which implies

$$
\left\|\left(\mathcal{M}_{m_{j}}-I\right) f\right\|_{\mathbf{L}^{\infty}} \leq C\|f\|_{\mathbf{H}^{\frac{1}{2}+s}}^{\frac{1}{1+2 s}}\left\|\left(\mathcal{M}_{m_{j}}-I\right) f\right\|_{\mathbf{L}^{2}}^{\frac{2 s}{1+2 s}} \leq C t^{-\frac{s}{2}}\|f\|_{\mathbf{H}^{\frac{1}{2}+s}} .
$$

Therefore

$$
\begin{aligned}
& \left\|R_{3, j}\right\|_{\mathbf{L}^{\infty}} \\
= & \left\|\left(\mathcal{M}_{m_{j}}-I\right) \frac{m_{j}}{t} F_{j}\left(D_{\frac{m_{j}}{m_{1}}} \mathcal{M}_{m_{1}}^{-1} D_{\frac{1}{m_{1}}}^{-1} u_{1}, D_{\frac{m_{j}}{m_{2}}} \mathcal{M}_{m_{2}}^{-1} D_{\frac{1}{m_{2}}}^{-1} u_{2}, D_{\frac{m_{j}}{m_{3}}} \mathcal{M}_{m_{3}}^{-1} D_{\frac{1}{m_{3}}}^{-1} u_{3}\right)\right\|_{\mathbf{L}^{\infty}} \\
\leq & C t^{-1-\frac{s}{2}}\left\|F_{j}\left(D_{\frac{m_{j}}{m_{1}}} \mathcal{M}_{m_{1}}^{-1} D_{\frac{1}{m_{1}}}^{-1} u_{1}, D_{\frac{m_{j}}{m_{2}}} \mathcal{M}_{m_{2}}^{-1} D_{\frac{1}{m_{2}}}^{-1} u_{2}, D_{\frac{m_{j}}{m_{3}}} \mathcal{M}_{m_{3}}^{-1} D_{\frac{1}{m_{3}}}^{-1} u_{3}\right)\right\|_{\mathbf{H}^{\frac{1}{2}+s}},
\end{aligned}
$$

where $F_{j}$ is a cubic nonlinearity which satisfies (1.2). Hence we have

$$
\left\|R_{3, j}\right\|_{\mathbf{L}^{\infty}} \leq C t^{-1-\frac{s}{2}} \sum_{j=1}^{3}\left\|U_{\frac{1}{m_{j}}}(-t) v_{j}\right\|_{\mathbf{H}^{0, \frac{1}{2}+s}}^{3}
$$

for $t \geq 1$. In the same way as above we obtain

$$
\left\|R_{4, j}\right\|_{\mathbf{L}^{\infty}} \leq C t^{-1-\frac{s}{2}} \sum_{j=1}^{3}\left\|U_{\frac{1}{m_{j}}}(-t) v_{j}\right\|_{\mathbf{H}^{0, \frac{1}{2}+s}}^{3}
$$

for $t \geq 1$. Combining all the inequalities obtained above, we have the lemma.

Lemma 2.3 Let $\left(v_{j}\right)_{j=1,2,3}$ be a solution of the system (1.1) satisfying (2.1) and $\frac{1}{2}<s<1$, then there exist $k_{j}>0$ for $j=1,2,3$ such that the estimate

$$
\begin{aligned}
& \frac{d}{d t} \sum_{j=1}^{3} k_{j}\left\|D_{\frac{1}{m_{j}}} \mathcal{F} U_{\frac{1}{m_{j}}}(-t) v_{j}\right\|_{\mathbf{L}^{\infty}} \\
\leq & C\left(\sum_{j=1}^{3} \varepsilon^{\frac{2}{3}\left(p_{j}+1\right)} t^{-\frac{p_{j}}{2}+\sqrt{\varepsilon}}+\sum_{j=1}^{3} \varepsilon^{\frac{2}{3}\left(p_{j}+1\right)} t^{-\frac{p_{j}}{2}-\frac{s}{2} p_{j}+\sqrt{\varepsilon}\left(p_{j}+1\right)}+\varepsilon^{2} t^{-1-\frac{s}{2}+3 \sqrt{\varepsilon}}\right)
\end{aligned}
$$

holds for any $t \in[1, T]$, where $u_{j}=D_{\frac{1}{m_{j}}} \mathcal{F} U_{\frac{1}{m_{j}}}(-t) v_{j}$. 
Proof (2.2) shows that

$$
i \partial_{t} u_{j}=\lambda_{j} D_{\frac{1}{m_{j}}} \mathcal{F} U_{\frac{1}{m_{j}}}(-t)\left|v_{j}\right|^{p_{j}} v_{j}+D_{\frac{1}{m_{j}}} \mathcal{F} U_{\frac{1}{m_{j}}}(-t) F_{j},
$$

where $u_{j}=D_{\frac{1}{m_{j}}} \mathcal{F} U_{\frac{1}{m_{j}}}(-t) v_{j}$. By $(1.2)$, we have

$$
i \partial_{t} u_{j}=\lambda_{j} D_{\frac{1}{m_{j}}} \mathcal{F} U_{\frac{1}{m_{j}}}(-t)\left|v_{j}\right|^{p_{j}} v_{j}+\frac{1}{t} F_{j}\left(u_{1}, u_{2}, u_{2}\right)+D_{\frac{1}{m_{j}}} \sum_{3}^{4} R_{h, j},
$$

From (1.11), we have

$$
\frac{d}{d t}\left(\sum_{j=1}^{3} k_{j}\left|u_{j}\right|\right) \leq C \sum_{j=1}^{3} \sum_{h=3}^{4}\left|R_{h, j}\right|+C \sum_{j=1}^{3}\left\|\mathcal{F} U_{\frac{1}{m_{j}}}(-t)\left|v_{j}\right|^{p_{j}} v_{j}\right\|_{\mathbf{L}^{\infty}},
$$

where $k_{j}>0$ for $j=1,2,3$. By Lemma 2.2 , we have

$$
\sum_{j=1}^{3} \sum_{h=3}^{4}\left|R_{h, j}\right| \leq C \varepsilon^{2} t^{-1-\frac{s}{2}+3 \sqrt{\varepsilon}}
$$

From (2.4) and Lemma 2.1 in [15], it follows that

$$
\begin{aligned}
& \sum_{j=1}^{3}\left\|\mathcal{F} U_{\frac{1}{m_{j}}}(-t)\left|v_{j}\right|^{p_{j}} v_{j}\right\|_{\mathbf{L}^{\infty}} \\
\leq & C \sum_{j=1}^{3}\left\|\mathcal{F} U_{\frac{1}{m_{j}}}(-t)\left|v_{j}\right|^{p_{j}} v_{j}\right\|_{\mathbf{H}^{\frac{1}{2}+s}}^{\frac{1}{1+2 s}}\left\|\mathcal{F} U_{\frac{1}{m_{j}}}(-t)\left|v_{j}\right|^{p_{j}} v_{j}\right\|_{\mathbf{L}^{2}}^{\frac{2 s}{1+2 s}} \\
\leq & C \sum_{j=1}^{3}\left(\left\|v_{j}\right\|_{\mathbf{L}^{\infty}}^{p_{j}}\left\|U_{\frac{1}{m_{j}}}(-t) v_{j}\right\|_{\mathbf{H}^{0, \frac{1}{2}+s}}\right)^{\frac{1}{1+2 s}}\left\|\left|v_{j}\right|^{p_{j}} v_{j}\right\|_{\mathbf{L}^{2}}^{\frac{2 s}{1+2 s}} \\
\leq & C \sum_{j=1}^{3}\left\|v_{j}\right\|_{\mathbf{L}^{\infty}}^{p_{j}}\left\|U_{\frac{1}{m_{j}}}(-t) v_{j}\right\|_{\mathbf{H}^{0, \frac{1}{2}+s}}^{\frac{1}{1+2 s}}\left\|v_{j}\right\|_{\mathbf{L}^{2}}^{\frac{2 s}{1+2 s}} \\
\leq & C \sum_{j=1}^{3}\left\|v_{j}\right\|_{\mathbf{L}^{\infty}}^{p_{j}}\left\|U_{\frac{1}{m_{j}}}(-t) v_{j}\right\|_{\mathbf{H}^{0, \frac{1}{2}+s}} .
\end{aligned}
$$

Since

we obtain

$$
\left\|v_{j}\right\|_{\mathbf{L}^{\infty}} \leq C t^{-\frac{1}{2}}\left\|\mathcal{F} U_{\frac{1}{m_{j}}}(-t) v_{j}\right\|_{\mathbf{L}^{\infty}}+C t^{-\frac{1}{2}-\frac{s}{2}}\left\|U_{\frac{1}{m_{j}}}(-t) v_{j}\right\|_{\mathbf{H}^{0, \frac{1}{2}+s}},
$$

$$
\sum_{j=1}^{3}\left\|\mathcal{F} U_{\frac{1}{m_{j}}}(-t)\left|v_{j}\right|^{p_{j}} v_{j}\right\|_{\mathbf{L}^{\infty}} \leq C \sum_{j=1}^{3} \varepsilon^{\frac{2}{3}\left(p_{j}+1\right)}\left(t^{-\frac{p_{j}}{2}+\sqrt{\varepsilon}}+t^{-\frac{p_{j}}{2}-\frac{s}{2} p_{j}+\sqrt{\varepsilon}\left(p_{j}+1\right)}\right) .
$$

This completes the proof of the lemma.

Lemma 2.4 Let $\left(v_{j}\right)_{j=1,2,3}$ be a solution of the system (1.1) satisfying (2.1). Then we have

$$
\begin{aligned}
\frac{d}{d t} \sum_{j=1}^{3}\left\|\left|J_{\frac{1}{m_{j}}}\right|^{\frac{1}{2}+s} v_{j}\right\| \leq & C\left(t^{-1+\sqrt{\varepsilon}}+t^{-1-s+3 \sqrt{\varepsilon}}\right) \varepsilon^{2} \\
& +C \sum_{j=1}^{3}\left(t^{-\frac{p_{j}}{2}+\sqrt{\varepsilon}}+t^{-\frac{p_{j}}{2}-\frac{p_{j}}{2} s+\left(p_{j}+1\right) \sqrt{\varepsilon}}\right) \varepsilon^{\frac{2}{3}\left(p_{j}+1\right)}
\end{aligned}
$$

for any $t \in[1, T]$. 


\section{Proof Let}

$$
\begin{aligned}
& N_{1}:=N_{1}\left(v_{1}, v_{2}, v_{3}\right)=\lambda_{1}\left|v_{1}\right|^{p_{1}} v_{1}+F_{1}, \\
& N_{2}:=N_{2}\left(v_{1}, v_{2}, v_{3}\right)=\lambda_{2}\left|v_{2}\right|^{p_{2}} v_{2}+F_{2}, \\
& N_{3}:=N_{3}\left(v_{1}, v_{2}, v_{3}\right)=\lambda_{3}\left|v_{3}\right|^{p_{3}} v_{3}+F_{3} .
\end{aligned}
$$

Since the commutation relation $\left[i \partial_{t}+\frac{1}{2 m_{j}} \partial_{x}^{2},\left|J_{\frac{1}{m_{j}}}\right|^{\gamma}\right]=0$ holds, we have the following result from the system (1.1)

$$
\left(i \partial_{t}+\frac{1}{2 m_{j}} \partial_{x}^{2}\right)\left|J_{\frac{1}{m_{j}}}\right|^{\gamma} v_{j}=\left|J_{\frac{1}{m_{j}}}\right|^{\gamma} N_{j}
$$

We multiply both sides of (2.8) by $\overline{\left|J_{\frac{1}{m_{j}}}\right|^{\gamma} v_{j}}$, integrate in space and take the imaginary parts to obtain

$$
\frac{d}{d t}\left\|\left|J_{\frac{1}{m_{j}}}\right|^{\gamma} v_{j}\right\|^{2}=2 \operatorname{Im} \int_{\mathbb{R}}\left|J_{\frac{1}{m_{j}}}\right|^{\gamma} N_{j} \cdot \overline{\left|J_{\frac{1}{m_{j}}}\right|^{\gamma} v_{j}} d x
$$

From $\left|J_{\frac{1}{m_{j}}}\right|^{\gamma}(t)=M^{-m_{j}}\left(-\frac{t^{2}}{m_{j}^{2}} \Delta\right)^{\frac{\gamma}{2}} M^{m_{j}}$ and the mass resonance condition (1.2), we have

$$
\left|J_{\frac{1}{m_{j}}}\right|^{\gamma} F_{j}\left(v_{1}, v_{2}, v_{3}\right)=M^{-m_{j}}\left(-\frac{t^{2}}{m_{j}^{2}} \Delta\right)^{\frac{\gamma}{2}} F_{j}\left(M^{m_{1}} v_{1}, M^{m_{2}} v_{2}, M^{m_{3}} v_{3}\right) .
$$

By Lemma 2.1 in [15] and (2.7) we get with $\gamma=\frac{1}{2}+s$

$$
\begin{aligned}
& \frac{d}{d t}\left\|\left|J_{\frac{1}{m_{j}}}\right|^{\gamma} v_{j}\right\| \\
\leq & C \sum_{j=1}^{3}\left(\left\|v_{j}\right\|_{\mathbf{L}^{\infty}}^{2}+\left\|v_{j}\right\|_{\mathbf{L}^{\infty}}^{p_{j}}\right) \sum_{j=1}^{3}\left\|\left|J_{\frac{1}{m_{j}}}\right|^{\gamma} v_{j}\right\| \\
\leq & C\left(t^{-1+\sqrt{\varepsilon}}+t^{-1-s+3 \sqrt{\varepsilon}}\right) \varepsilon^{2} \\
& +C \sum_{j=1}^{3}\left(t^{-\frac{p_{j}}{2}+\sqrt{\varepsilon}}+t^{-\frac{p_{j}}{2}-\frac{p_{j}}{2} s+\left(p_{j}+1\right) \sqrt{\varepsilon}}\right) \varepsilon^{\frac{2}{3}\left(p_{j}+1\right)} .
\end{aligned}
$$

This completes the proof of the lemma.

By Lemmas 2.3, 2.4 we have

$$
\sum_{j=1}^{3} k_{j}\left\|D_{\frac{1}{m_{j}}} \mathcal{F} U_{\frac{1}{m_{j}}}(-t) v_{j}\right\|_{\mathbf{L}^{\infty}} \leq C \varepsilon+\sum_{j=1}^{3} \varepsilon^{\frac{2}{3}\left(p_{j}+1\right)}+\varepsilon^{2}<C \varepsilon
$$

and

$$
\sum_{j=1}^{3}\left\|U_{\frac{1}{m_{j}}}(-t) v_{j}\right\|_{\mathbf{H}^{0, \frac{1}{2}+s}} \leq 2 \varepsilon+\varepsilon^{2} t^{\sqrt{\varepsilon}}+\sum_{j=1}^{3} \varepsilon^{\frac{2}{3}\left(p_{j}+1\right)}<C \varepsilon t^{\sqrt{\varepsilon}}
$$

since $s>0, p_{j}>2$, there exists $\varepsilon>0$ such that $\frac{s}{2}>3 \sqrt{\varepsilon}, \frac{p_{j}-2}{2}>\sqrt{\varepsilon}$. This is the desired contradiction.

Therefore, we have a unique global solution of the system (1.1) satisfying

$$
\sup _{t \in[1, T]} \sum_{j=1}^{3}\left((1+t)^{-\sqrt{\varepsilon}}\left\|U_{\frac{1}{m_{j}}}(-t) v_{j}\right\|_{\mathbf{H}^{0, \frac{1}{2}+s}}+\left\|D_{\frac{1}{m_{j}}} \mathcal{F} U_{\frac{1}{m_{j}}}(-t) v_{j}\right\|_{\mathbf{L}^{\infty}}\right)<\varepsilon^{\frac{2}{3}}
$$

for any $T>1$. Time decay estimates of solutions to the system (1.1) comes from (2.7). Namely we have

$$
\left\|v_{j}\right\|_{\mathbf{L}^{\infty}} \leq C t^{-\frac{1}{2}} \varepsilon^{\frac{2}{3}}+C t^{-\frac{1}{2}-\frac{s}{2}+\sqrt{\varepsilon}} \varepsilon^{\frac{2}{3}} .
$$

This completes the proof of Theorem 1.1. 


\section{Proof of Theorems 1.2 and 1.3}

We define

$$
\|v\|_{X_{T}}=\sup _{t \in[0, T]} \sum_{j=1}^{3}\left((1+t)^{-\sqrt{\varepsilon}}\left\|U_{\frac{1}{m_{j}}}(-t) v_{j}\right\|_{\mathbf{H}^{0, \frac{1}{2}+s}}+(1+t)^{\frac{1}{p}-\frac{1}{2}}\left\|D_{\frac{1}{m_{j}}} \mathcal{F} U_{\frac{1}{m_{j}}}(-t) v_{j}\right\|_{\mathbf{L}^{\infty}}\right)
$$

where $p=p_{j}, j=1,2,3, \frac{1}{2}<s \leq 1, T>0$ and $\varepsilon>0$ is sufficiently small. In the same way as in the proof of Theorem 1.1, we may assume that

$$
\sum_{j=1}^{3}\left(\left\|U_{\frac{1}{m_{j}}}(-1) v_{j}(1)\right\|_{\mathbf{H}^{0, \frac{1}{2}+s}}+\left\|D_{\frac{1}{m_{j}}} \mathcal{F} U_{\frac{1}{m_{j}}}(-1) v_{j}(1)\right\|_{\mathbf{L}^{\infty}}\right) \leq 2 \varepsilon .
$$

By the contradiction method, we prove for any $T>1$ the estimate

$$
\sup _{t \in[1, T]} \sum_{j=1}^{3}\left(t^{-\sqrt{\varepsilon}}\left\|U_{\frac{1}{m_{j}}}(-t) v_{j}\right\|_{\mathbf{H}^{0, \frac{1}{2}+s}}+t^{\frac{1}{p}-\frac{1}{2}}\left\|D_{\frac{1}{m_{j}}} \mathcal{F} U_{\frac{1}{m_{j}}}(-t) v_{j}\right\|_{\mathbf{L}^{\infty}}\right)<\varepsilon^{p-1}
$$

holds. We assume that there exists a time $T$ such that

$$
\sup _{t \in[1, T]} \sum_{j=1}^{3}\left(t^{-\sqrt{\varepsilon}}\left\|U_{\frac{1}{m_{j}}}(-t) v_{j}\right\|_{\mathbf{H}^{0, \frac{1}{2}+s}}+t^{\frac{1}{p}-\frac{1}{2}}\left\|D_{\frac{1}{m_{j}}} \mathcal{F} U_{\frac{1}{m_{j}}}(-t) v_{j}\right\|_{\mathbf{L}}\right) \leq \varepsilon^{p-1} .
$$

We derive the desired contradiction to extend the proof in [13] to subcritical nonlinearities.

\subsection{Proof of Theorem 1.2}

We have by $(2.3)$

$$
\begin{aligned}
& \frac{d}{d t} f_{j}+\left|\operatorname{Im} \lambda_{j}\right| t^{-\frac{p}{2}} f_{j}^{p+1} \\
\leq & C t^{-1}\left|F_{j}\left(u_{1}, u_{2}, u_{3}\right)\right|+C\left|D_{\frac{1}{m_{j}}} \sum_{h=1}^{4} R_{h, j}\right|,
\end{aligned}
$$

where $f_{j}=\left|u_{j}\right|=\left|D_{\frac{1}{m_{j}}} \mathcal{F} U_{\frac{1}{m_{j}}}(-t) v_{j}\right|$. Let $g_{j}$ be the positive solution of

$$
\frac{d}{d t} g_{j}+\left|\operatorname{Im} \lambda_{j}\right| t^{-\frac{p}{2}} g_{j}^{p+1}=0, g_{j}(1)=\left|D_{\frac{1}{m_{j}}} \mathcal{F} U_{\frac{1}{m_{j}}}(-1) v_{j}(1)\right| .
$$

We may assume that $\varepsilon \leq g_{j}(1) \leq 2 \varepsilon$ by the local existence result in Lemma 2.1. Explicit solution of (3.2) is given by

$$
g_{j}(t)=\frac{g_{j}(1)}{\left(1+\frac{2 p\left|\operatorname{Im} \lambda_{j}\right|}{2-p} g_{j}(1)^{p}\left(t^{1-\frac{p}{2}}-1\right)\right)^{\frac{1}{p}}} .
$$

The strong dissipation assumption (1.14) implies that (3.1) is valid if we replace $\left|\operatorname{Im} \lambda_{j}\right|$ by $\frac{1}{\varepsilon \sqrt{\varepsilon}}$. To keep $g_{j}(t)$ small enough, we use $p=2-\sqrt{\varepsilon}$ and explicit solution of (3.3) with $\frac{1}{\varepsilon \sqrt{\varepsilon}}=\left|\operatorname{Im} \lambda_{j}\right|$. Namely,

$$
g_{j}(t)=\frac{g_{j}(1)}{\left(1+\frac{2 p}{\varepsilon^{2}} g_{j}(1)^{p}\left(t^{1-\frac{p}{2}}-1\right)\right)^{\frac{1}{p}}} .
$$

Then we find that there exist positive constants $C_{1}, C_{2}$ such that

$$
C_{2} \varepsilon^{\frac{2}{p}} t^{-\frac{1}{p}+\frac{1}{2}} \leq g_{j}(t) \leq C_{1} \varepsilon^{\frac{2}{p}} t^{-\frac{1}{p}+\frac{1}{2}}
$$


for a large $t$. Multiplying both sides of (3.1) by $g_{j}^{-p-1}$, we have

$$
\frac{d}{d t}\left(g_{j}^{-p-1} f_{j}\right) \leq t^{-\frac{p}{2}} \frac{1}{\varepsilon \sqrt{\varepsilon}}\left((p+1) g_{j}^{-1} f_{j}-g_{j}^{-p-1} f_{j}^{p_{j}+1}\right)+C g_{j}^{-p-1} R_{j},
$$

where

$$
R_{j}=C t^{-1}\left|F_{j}\left(u_{1}, u_{2}, u_{3}\right)\right|+C\left|D_{\frac{1}{m_{j}}} \sum_{h=1}^{4} R_{h, j}\right| .
$$

In the same way as in the proof of Lemma 2.2, we get

$$
\left|D_{\frac{1}{m_{j}}} \sum_{h=1}^{4} R_{h, j}\right| \leq C \varepsilon^{3(p-1)} t^{-1-\frac{s}{2}+3 \sqrt{\varepsilon}}+C \varepsilon^{-\frac{3}{2}} \varepsilon^{(p-1)(p+1)} t^{-\frac{p}{2}-\frac{s}{2}+(p+1) \sqrt{\varepsilon}} .
$$

Hence we get

$$
\begin{aligned}
R= & \sum_{j=1}^{3} R_{j} \leq C \varepsilon^{3(p-1)} t^{-1+3\left(\frac{1}{2}-\frac{1}{p}\right)}+C \varepsilon^{3(p-1)} t^{-1-\frac{s}{2}+3 \sqrt{\varepsilon}} \\
& +C \varepsilon^{-\frac{3}{2}} \varepsilon^{(p-1)(p+1)} t^{-\frac{p}{2}-\frac{s}{2}+(p+1) \sqrt{\varepsilon}} .
\end{aligned}
$$

The second and third terms of the right hand side of (3.7) are considered as remainder terms. We note here that the third term has $\varepsilon^{-\frac{3}{2}}$ since the condition (1.14). By the Young inequality $|a||b| \leq \frac{1}{p}|a|^{p}+\frac{1}{q}|b|^{q}$ with $\frac{1}{p}+\frac{1}{q}=1$, we get

$$
(p+1) g_{j}^{-1} f_{j}=\left((p+1)^{\frac{1}{p+1}} g_{j}^{-1} f_{j}\right)(p+1)^{\frac{p}{p+1}} \leq g_{j}^{-p-1} f_{j}^{p+1}+p .
$$

Hence

$$
\frac{d}{d t}\left(g_{j}^{-p_{j}-1} f_{j}\right) \leq p \frac{1}{\varepsilon \sqrt{\varepsilon}} t^{-\frac{p}{2}}+C g_{j}^{-p-1} R .
$$

Integrating in time, we obtain by (3.5)

$$
\begin{aligned}
f_{j}(t) \leq & g_{j}^{-p-1}(1) f_{j}(1) g_{j}^{p+1}(t)+\frac{2 p}{2-p} g_{j}^{p+1}(t) \frac{1}{\varepsilon \sqrt{\varepsilon}}\left(t^{1-\frac{p}{2}}-1\right) \\
& +C g_{j}^{p+1}(t) \int_{1}^{t} g_{j}^{-p-1}(\tau) R(\tau) d \tau \\
\leq & C \varepsilon^{2+\frac{2}{p}-p} t^{\left(\frac{1}{2}-\frac{1}{p}\right)(p+1)}+C \varepsilon^{\frac{2}{p}} t^{\left(\frac{1}{2}-\frac{1}{p}\right)(p+1)} t^{1-\frac{p}{2}} \\
& +C t^{\left(\frac{1}{2}-\frac{1}{p}\right)(p+1)} \int_{1}^{t} \tau^{-\left(\frac{1}{2}-\frac{1}{p}\right)(p+1)} R(\tau) d \tau .
\end{aligned}
$$

We apply (3.7) to the right hand side of the above to get

$$
\begin{aligned}
f_{j}(t) \leq & C \varepsilon^{2+\frac{2}{p}-p} t^{\left(\frac{1}{2}-\frac{1}{p}\right)(p+1)}+C \varepsilon^{\frac{2}{p}} t^{\left(\frac{1}{2}-\frac{1}{p}\right)(p+1)} t^{1-\frac{p}{2}} \\
& +C \varepsilon^{3(p-1)} t^{\left(\frac{1}{2}-\frac{1}{p}\right)(p+1)} \int_{1}^{t} \tau^{-\left(\frac{1}{2}-\frac{1}{p}\right)(p+1)+\left(\frac{1}{2}-\frac{3}{p}\right)} d \tau,
\end{aligned}
$$

if we take a suitable $s$. Since

$$
-\left(\frac{1}{2}-\frac{1}{p}\right)(p+1)+\left(\frac{1}{2}-\frac{3}{p}\right)=-1-\frac{\varepsilon}{2(2-\sqrt{\varepsilon})},
$$

we get

$$
\int_{1}^{t} \tau^{-\left(\frac{1}{2}-\frac{1}{p}\right)(p+1)+\frac{1}{2}-\frac{3}{p}} d \tau \leq C \varepsilon^{-1}
$$


Hence

$$
f_{j}(t) \leq C \varepsilon t^{\left(\frac{1}{2}-\frac{1}{p}\right)}+C \varepsilon^{3 p-3} \varepsilon^{-1} t^{\left(\frac{1}{2}-\frac{1}{p}\right)(p+1)},
$$

from which it follows that

$$
t^{\frac{1}{p}-\frac{1}{2}}\left\|D_{\frac{1}{m_{j}}} \mathcal{F} U_{\frac{1}{m_{j}}}(-t) v_{j}\right\|_{\mathbf{L}^{\infty}} \leq C \varepsilon+C \varepsilon^{3 p-4} \leq C \varepsilon
$$

where $p \geq \frac{5}{3}$. By $(2.7)$, we obtain

$$
\left\|v_{j}\right\|_{\mathbf{L}^{\infty}} \leq C \varepsilon t^{-\frac{1}{p}}+C \varepsilon^{p-1} t^{-\frac{1}{2}-\frac{s}{2}+\sqrt{\varepsilon}} \leq C \varepsilon^{p-1} t^{-\frac{1}{p}}
$$

if $-\frac{s}{2}+\sqrt{\varepsilon}<\frac{1}{2}-\frac{1}{p}$. In the same way as in the proof of (2.9) with $\gamma=\frac{1}{2}+s$

$$
\begin{aligned}
& \frac{d}{d t} \sum_{j=1}^{3}\left\|\left|J_{\frac{1}{m_{j}}}\right|^{\gamma} v_{j}\right\| \\
\leq & C \sum_{j=1}^{3}\left\|v_{j}\right\|_{\mathbf{L}^{\infty}}^{p_{j}}\left\|\left|J_{\frac{1}{m_{j}}}\right|^{\gamma} v_{j}\right\|+\sum_{j=1}^{3}\left\|v_{j}\right\|_{\mathbf{L}^{\infty}}^{2} \sum_{j=1}^{3}\left\|\left|J_{\frac{1}{m_{j}}}\right|^{\gamma} v_{j}\right\| \\
\leq & C\left(t^{-1} \varepsilon^{(p-1) p} \sum_{j=1}^{3}\left\|\left|J_{\frac{1}{m_{j}}}\right|^{\gamma} v_{j}\right\|+\varepsilon^{2(p-1)} t^{-\frac{2}{p}} \sum_{j=1}^{3}\left\|\left|J_{\frac{1}{m_{j}}}\right|^{\gamma} v_{j}\right\|\right) \\
\leq & C \varepsilon^{(p-1) p}\left(t^{-1}+t^{-\frac{2}{p}}\right) \sum_{j=1}^{3}\left\|\left|J_{\frac{1}{m_{j}}}\right|^{\gamma} v_{j}\right\| \\
\leq & C \varepsilon^{(p-1) p} t^{-1} \sum_{j=1}^{3}\left\|\left|J_{\frac{1}{m_{j}}}\right|^{\gamma} v_{j}\right\| .
\end{aligned}
$$

Gronwall's inequality says that

$$
\sum_{j=1}^{3}\left\|\left|J_{\frac{1}{m_{j}}}\right|^{\gamma} v_{j}\right\| \leq C \varepsilon e^{C \int_{1}^{t} \varepsilon^{(p-1) p} \tau^{-1} d \tau}=C \varepsilon t^{C \varepsilon^{(p-1) p}} \leq C \varepsilon t^{\sqrt{\varepsilon}}
$$

Hence

$$
t^{\frac{1}{p}-\frac{1}{2}}\left\|D_{\frac{1}{m_{j}}} \mathcal{F} U_{\frac{1}{m_{j}}}(-t) v_{j}\right\|_{\mathbf{L}^{\infty}}+t^{-\sqrt{\varepsilon}}\left\|\left|J_{\frac{1}{m_{j}}}\right|^{\gamma} v_{j}\right\| \leq C \varepsilon<\varepsilon^{p-1} .
$$

This is the desired contradiction. Time decay of solutions is obtained by (3.9). This completes the proof of Theorem 1.2.

\subsection{Proof of Theorem $\mathbf{1 . 3}$}

By the condition of (1.11), we have in the same way as in the proof of (3.1)

$$
\sum_{j=1}^{3} k_{j}\left(\frac{d}{d t} f_{j}+\left|\operatorname{Im} \lambda_{j}\right| t^{-\frac{p}{2}} f_{j}^{p+1}\right) \leq Q
$$

where by (3.6)

$$
\begin{aligned}
Q & =C\left|\sum_{j=1}^{3} k_{j} D_{\frac{1}{m_{j}}} \sum_{h=1}^{4} R_{h, j}\right| \\
& \leq C \varepsilon^{3(p-1)} t^{-1-\frac{s}{2}+3 \sqrt{\varepsilon}}+C \varepsilon^{(p-1)(p+1)} t^{-\frac{p}{2}-\frac{s}{2}+(p+1) \sqrt{\varepsilon}} .
\end{aligned}
$$


We put $f=\sum_{j=1}^{3} k_{j} f_{j}$, then there exists a positive constant $k$ such that

$$
\frac{d}{d t} f+k t^{-\frac{p}{2}} f^{p+1} \leq C Q
$$

Let $g$ be the positive solution of

$$
\frac{d}{d t} g+k t^{-\frac{p}{2}} g^{p+1}=0, g(1)=\sum_{j=1}^{3} k_{j}\left|D_{\frac{1}{m_{j}}} \mathcal{F} U_{\frac{1}{m_{j}}}(-1) v_{j}(1)\right|
$$

Explicit solution of (3.12) is given by

$$
g(t)=\frac{g(1)}{\left(1+\frac{2 p k}{2-p} g(1)^{p}\left(t^{1-\frac{p}{2}}-1\right)\right)^{\frac{1}{p}}} .
$$

If $p=2-\varepsilon^{p}$, then we find that there exist positive constants $C_{3}, C_{4}$ such that

$$
C_{3} \varepsilon t^{-\frac{1}{p}+\frac{1}{2}} \leq g(t) \leq C_{4} \varepsilon t^{-\frac{1}{p}+\frac{1}{2}}
$$

In the same way as in the proof of $(3.8)$

$$
\begin{aligned}
f(t) \leq & g^{-p-1}(1) f(1) g^{p+1}(t)+\frac{2 p}{2-p} g^{p+1}(t) k\left(t^{1-\frac{p}{2}}-1\right) \\
& +C g^{p+1}(t) \int_{1}^{t} g^{-p-1}(\tau) Q(\tau) d \tau \\
\leq & C \varepsilon t^{\left(\frac{1}{2}-\frac{1}{p}\right)(p+1)}+C \varepsilon t^{\left(\frac{1}{2}-\frac{1}{p}\right)(p+1)} t^{1-\frac{p}{2}}
\end{aligned}
$$

which implies that

$$
t^{\frac{1}{p}-\frac{1}{2}}\left\|D_{\frac{1}{m_{j}}} \mathcal{F} U_{\frac{1}{m_{j}}}(-t) v_{j}\right\|_{\mathbf{L}^{\infty}} \leq C \varepsilon .
$$

The rest of the proof is the same as in the proof of Theorem 1.2, and so we omit it.

Acknowledgments. The authors would like to thank Professor Nakao Hayashi for many useful comments. The work is partially supported by NNSFC Grant No.11461074.

\section{References}

1. G. P. Agrawal, "Nonlinear Fiber Optics," Academic Press, 1995.

2. J. E. Barab, "Nonexistence of asymptotically free solutions for a nonlinear Schrödinger equation," Journal of Mathematical Physics, vol. 25, no. 11, pp. 3270-3273, 1984.

3. R. Carles, "Geometric optics and long range scattering for one-dimensional nonlinear Schrödinger equations," Communications in Mathematical Physics, vol. 220, pp. 41-67, 2001.

4. T.Cazenave and F. B. Weissler, "The Cauchy problem for the critical nonlinear Schrödinger equation in $\mathbf{H}^{\mathbf{s}}$," Nonlinear Analysis, vol. 14, no. 10, pp. 807-836, 1990.

5. M. Colin and T. Colin, "On a quasilinear Zakharov system describing laser-plasma interactions," Differential and Integral Equations, vol. 17, no. 3-4, pp. 297-330, 2004.

6. N. Hayashi and P. I. Naumkin, "Asymptotics for large time of solutions to the nonlinear Schrödinger and Hartree equations," American Journal of Mathematics, vol. 120, no. 2, pp. 369-389, 1998.

7. N. Hayashi and P.I. Naumkin, "Domain and range of the modified wave operator for Schrödinger equations with a critical nonlinearity," Communications in Mathematical Physics, vol. 267, pp. 477-492, 2006.

8. N. Hayashi, C. Li and P. I. Naumkin, "On a system of nonlinear Schrödinger equations in 2d," Differential and Integral Equations, vol. 24, no. 5-6, pp. 417-434, 2011.

9. N. Hayashi, C. Li and P. I. Naumkin, "Nonlinear Schrödinger systems in 2d with nondecaying final data," Journal of Differential Equations, vol. 260, pp. 1472-1495, 2016. 
10. N. Hayashi and T. Ozawa, "Scattering theory in weighted $\mathbf{L}^{2}\left(\mathbb{R}^{n}\right)$ spaces for some Schrödinger equations," Annales De l'Institut Henri Poincaŕe Physique Théorique, vol. 48, pp. 17-37, 1988.

11. N. Hayashi, T. Ozawa and K. Tanaka, "On a system of nonlinear Schrödinger equations with quadratic interaction," Annales De l'Institut Henri Poincaŕe (C) Non Linear Analysis, vol. 30, pp. 661-690, 2013.

12. G. Jin, Y. Jin and C. Li, "The initial value problem for nonlinear Schödinger equations with a dissipative nonlinearity in one space dimension," Journal of Evolution Equations, vol. 16, no. 4, pp. 983-995, 2016.

13. S. Katayama, C. Li and H. Sunagawa, "A remark on decay rates of solutions for a system of quadratic nonlinear Schrödinger equations in 2D, " Differential and Integral Equations, vol. 27, no. 3-4, pp. 301-312, 2014.

14. D. Kim, "A note on decay rates of solutions to a system of cubic nonlinear Schrödinger equations in one space dimension," Asymptotic Analysis, vol. 98, no. 1-2, pp. 79-90, 2016.

15. N. Kita and A. Shimomura, "Asymptotic behavior of solutions to Schrödinger equations with a subcritical dissipative nonlinearity," Journal of Differential Equations, vol. 242, pp. 192-210, 2007.

16. N. Kita and A. Shimomura, "Large time behavior of solutions to Schrödinger equations with a dissipative nonlinearity for arbitrarily large initial data," Journal of the Mathematical Society of Japan, vol. 61, no.1, pp. 39-64, 2009.

17. C. Li, "Decay of solutions for a system of nonlinear Schrödinger equations in 2D," Discrete and Continuous Dynamical Systems, vol. 32, no.12, pp. 4265-4285, 2012.

18. C. Li and H. Sunagawa, "On Schrödinger systems with cubic dissipative nonlinearities of derivative type," Nonlinearity, vol. 29, pp. 1537-1563, 2016.

19. X. Lü, W. Ma, J. Yu and C. Khalique, "Solitary waves with the Madelung fluid description: A generalized derivative nonlinear Schrödinger equation," Communications in Nonlinear Science and Numerical Simulation, vol. 31, no.1-3, pp. 40-46, 2016.

20. X. Lü and F. Lin, "Soliton excitations and shape-changing collisions in alpha helical proteins with interspine coupling at higher order," Communications in Nonlinear Science and Numerical Simulation, vol. 32, pp. 241-261, 2016.

21. T. Ogawa and K. Uriya, "Final state problem for a quadratic nonlinear Schrödinger system in two space dimensions with mass resonance," Journal of Differential Equations, vol. 258, pp. 483-503, 2015.

22. T. Ozawa, "Long range scattering for nonlinear Schrödinger equations in one space dimension," Communications in Mathematical Physics, vol. 139, pp. 479-493, 1991.

23. Y. Sagawa and H. Sunagawa, "The lifespan of small solutions to cubic derivative nonlinear Schrödinger equations in one space dimension," Discrete and Continuous Dynamical systems, vol. 36, pp. 5743-5761, 2016.

24. A. Shimomura, "Asymptotic behavior of solutions for Schrödinger equations with dissipative nonlinearities," Communications in Partial Differential Equations, vol. 31, pp. 1407-1423, 2006.

25. Y. Tsutsumi and K. Yajima, "The asymptotic behavior of nonlinear Schrödinger equations," Bulletin of the American Mathematical Society, vol. 11, no.1, pp. 186-188, 1984. 